\title{
Practicing Defensive Medicine Benefits No one
}

\begin{abstract}
Background: More doctors are practicing defensive medicine, which may have impact on patient care. This paper explores the consequences and reasons, for the widespread trend, and recommendations for patient focussed clinical care.
\end{abstract}

Objective: Malpractice suit is the most scarring ordeal, which a physician can undergo both emotionally and financially. Excessive investigations may be counterproductive. Avoiding certain procedures or patients, for the fear of adverse outcome can be hazardous.

There is a need for a better, discerning and judicious system, to minimise the trauma to the largely conscientious and dedicated medical profession.

\section{Discussion}

How and when the term defensive medicine started, is difficult to trace. However over the past few decades, it seems to be a well-known practice in industrialised countries. It may have been due to increasing complaints against doctors heralding potential litigation and malpractice suit.

Most doctors do not take the privilege of the trust of their patients lightly, and respond to it with utmost sincerity.

Keywords

Defensive medicine; Medical regulator; medical litigation

\section{Background}

Medical profession by its nature has within it, the realm of inexhaustible agenda of self-improvement, and life-long learning. It is further reinforced by the cultural norm of professional environment. Nevertheless there can be unscrupulous doctors, to smear the image. As human health remains the highest priority for each and everyone, there is no room for complacency, when dealing with someone else's health. To ensure that all the doctors remain on the path of near perfection of their role, a regulatory body is essential, to oversee and objectively evaluate the services we provide, and its outcome.

\section{Role of regulatory authorities}

In understanding defensive medicine, exploring its possible reason is indispensible. In some countries like Australia, Health Practitioners Regulating Authority's performance and methods have been extensively criticised.[1] In 2017, the senate committee in considering the issue of mandatory reporting concluded, that doctors lost confidence in the board's administered processes.[2] Although patient health is indisputable and equally valued by doctors, regulatory body's
Shailja Chaturvedi FRPsy ANZ

Psychiatrist, New South Wales, Australia

Shail150@gmail.com

Cite as: Chaturvedi, S. (2021) Practicing defensive medicine benefits no one. Sushruta J Health Pol \& Opin Vol 14; Issue 2: article 5, p1-4 DOI https://doi.org/10.38192/14. $\underline{2.5}$

Article Information

Submitted 3.6.21

Published 5.6.21

ISSN 2732-5164 (Online) ISSN 2732-5156 (Print)

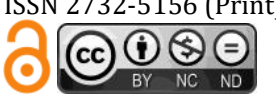


actions against doctors profoundly jeopardise their life. Doctors are mindful that ethics, human rights and rules must dictate their professional behaviour and patient safety and adhering to best practice and quality of care, should be reflected in their practice, as a competent doctor.

The regulating body must remain sensitive and aware of many challenges and situations a doctor must overcome, to provide ethical, appropriate and best possible service to their patients without contravening human rights. [3] Its investigations must not be driven by the complaints alone without validating it by collaborative evidence. It must take notice of weak as well as strong evidence of defence, against the alleged misadventure and must investigate the complaint beyond reasonable doubt to justify the conviction. It should not use civil court's balance of probability in judging the doctor, which is 50:50. Presuming unbiased approach, it will convict the innocent up to $49 \%$ of the time. The consequence of this lottery for the accused but innocent doctor can be devastating. [4] Medical Boards should have obligatory duty of care to the subjects of its investigations bound by the rules of evidence and fairness. Threat of disciplinary actions and even deregistration, contravenes the democratic rights of free speech, in discussing controversial issues impacting on human health. The best and only way to protect the public from medical misadventure is necessarily not the punishment of the doctor. Undoubtedly there are incompetent and dangerous doctors who need formal control but it is separate from prevention and effective public protection. [5]

\section{Defensive Medical Practice}

Between $60-90 \%$ in US and $78 \%$ physicians in UK, practice defensive medicine, ordering excessive procedures, tests and referrals for opinions, to minimize the threat of malpractice. [6] Despite widespread agreement that doctors practice defensive medicine to avoid malpractice liability, there is dearth of studies to explore it, for justifying increased resources to minimize the liability. In Australia $60 \%$ doctors engage in defensive practice, and $60-70 \%$ made practice changes due to medico-legal concerns [7] resulting in unnecessary care. Defensive medicine makes a large contribution to the health care cost in the tune of about 34\%.

Interpersonal factors like doctor-patient communication may be, one of the factors behind patients' dissatisfaction. The argument for defensive medicine is, that it improves quality of care. However the primary role of defensive medicine is not for the patient, but to prevent a malpractice suit, should a problem occur. There is some evidence that doctors, who ordered more tests, were substantially less likely to be sued by angry patients.[6]

One of the other ways to minimize the legal implications is by avoiding complex and high- risk patients and procedures and selecting a specialty with lesser risk of malpractice suits and better patient outcome. This results in avoiding certain invasive procedures, depriving the patient, who may need their skills most. This is more relevant in psychiatry where apart from litigation; emotional impact of patient outcome can encourage defensive medicine. Emotionally charged patients with untoward outcome like worsening of illness, aggression or suicide risk may be deprived of best psychiatric care. Diagnostic dilemma due to imprecise evidence and ambiguity in psychiatry is likely to impact on the treatment and the outcome. Involuntary treatment as a safeguard may be interpreted as overzealous, overriding human rights of the patient, and doing less can be seen as negligent by the authorities. Coercive legislation, human rights of the patient and defensive medicine often present a dilemma for a doctor.

Defensive medicine drives low value care with harm to the patient due to unnecessary investigations like body invasion through radiation etc. It violates justice and fiduciary obligations, undermining the trust in the profession. It is a challenge to medical ethics and professionalism. In some countries, law requires all health practitioners to report all notifiable conducts, which come to their attention to the regulating authority. It may be far reaching as it applies to peers, treating physician, employer and the education provider. As much as it has addressed the willingness to deal with legitimate concerns, it has created unproductive culture of fear, blame and vexatious reporting. [8]

"Being under investigation is a suicide risk-factor for doctors" was the subject of the poll conducted by Medical Journal of Australia in 2018. 74\% respondents strongly agreed, 23\% agreed and $1 \%$ disagreed. The disciplinary procedures have profound and serious consequences on health, personal life and professional functioning of the doctor specially, if the complaints were vexatious. [9-11] Fortunately majority of doctors will never be under investigation, but every doctor lives with this fear. Complaints against doctors are not necessarily made due to misadventure. It may be to 
blame the doctor, to deal with guilt, anger, sense of revenge, or even losing control over a recovered patient. [12]

A good example of it communicated to me, was a complaint made to the Medical Board by a relative. As the patient who served as a scapegoat, by remaining in dependent and submissive role, started to get better regaining confidence and autonomy, the family balance of coercive control was shaken. Patient's emerging confidence was misconstrued as aggression and grandiosity, for which the doctor was blamed and reported. The Board's investigations were based on the complaint alone, with no reference to any collateral information from the multi-disciplinary team, human rights [7] or the view of the patient who was enjoying the recovery and her independence. The subsequent series of performance reviews of the doctor were to validate the initial complaint much to the demise of a successful career.

Medical practice based on fear of legal liability rather than patient's interest is both dangerous and costly. It generates anger, guilt, shame and depression, undermining the trust and goodwill towards patients. [13-15] Doctors may relent to active treatment demands by the patients and the families without the considerations of the risks involved like injuries, complications or death, for the fear of complaints. [16]

\section{Prevention}

Defensive medicine may include, unconditionally complying with patient's wishes, feeling powerless, emotionally distressed and negatively towards the complainant and the regulatory authority. Prolonged duration and unpredictability of the procedure in dealing with the complaint by the regulatory authority, their managerial incompetence, poor communication and possible breach of confidentiality are likely to be biased in favour of the complainant.

- There should be open dialogue with the complainant.

- The procedure should be simplified and time limited.

- It should be more transparent with comprehensive evidence to test the authenticity of the complaint.

- Complexity of medicine, fallibility of doctors, disparity between social expectations of care and realistic fulfilment of it, must be discussed with the complainant.
- Perceived social culture of blame must be equally appreciated and understood, by the complainant and the doctor.

- Complaints must be resolved rapidly.

- Frivolous and vexatious complaints should be identified.

- The regulatory authority must acknowledge socio-political context of the delivery of care, as well as, the impact of complaints on the doctor.

- Decriminalisation of medical errors, increased time directly spent with patient, importance of clinical reasoning and institutional support must be pursued as achievable goals.

- The accused physician's basis for particular clinical decision should be considered in the light of conventional and current best practice (which may not necessarily be the view of the Board appointed reviewer).

\section{Conclusion}

Concern for potential litigation may be the common reason for professional dissatisfaction amongst doctors. [17] The competing interest of self- protection and patient's best interest may result in conflicting outcome. Poor processing, prolonged time scale and vexatious use of complaints is associated with diminished psychological welfare and increased defensive medicine. $40 \%$ malpractice cases are found to be without any medical error though the journey through the investigations can break the doctor to fail all the performance reviews conducted under extreme mental agony. Physicians therefore err on the side of caution and practice defensive medicine in an attempt to avoid an unfortunate outcome.

The regulatory body should not be allowed to become oppressive bureaucrat through the arbitrary use and abuse of its power. Changing its policy of focus on the allegations, it must endeavour to accomplish transparent, unbiased and justified outcome of comprehensive enquiry, with clear perception of the sequence of the events. The doctor must not pay the price for the inconsistencies in the health care system, which may be the root cause.

Better ways, of separating healthy from bad apples, beyond reasonable doubt, to prevent the practice of defensive medicine must be devised. Providing ethical care by a conscientious doctor can be undermined by practice of defensive medicine. 
References

1 Ford N. The elephant in the consulting room. Aust N Z J Psychiatry 2019;53:809-10. doi:10.1177/0004867419844600

2 Complaints mechanism administered under the Health Practitioner Regulation National Law - Parliament of Australia. https://www.aph.gov.au/Parliamentary_Busin ess/Committees/Senate/Community_Affairs/C omplaintsMechanism (accessed 5 Jun 2021).

3 Mental Health Declaration of Human Rights. Citizens Commission on Human Rights, CCHR - Nonprofit Mental Health Watchdog. 1AD.https://www.cchr.org/about-us/mentalhealth-declaration-of-human-rights.html (accessed 5 Jun 2021).

4 Australia's Star Chamber. Australian Medical Association (NSW).

2018.https://www.amansw.com.au/australias -star-chamber/ (accessed 5 Jun 2021).

5 Finlay AJF, Stewart CL, Parker M. Open disclosure: ethical, professional and legal obligations, and the way forward for regulation. The Medical Journal of Australia 2013;198:445-8. doi:10.5694/mja12.10734

6 Physician spending and subsequent risk of malpractice claims: observational study | The BMJ.

https://www.bmj.com/content/351/bmj.h55 16.long (accessed 5 Jun 2021).

$7 \quad$ Perceived practice change in Australian doctors as a result of medicolegal concerns | The Medical Journal of Australia. https://www.mja.com.au/journal/2010/193/ $10 /$ perceived-practice-change-australiandoctors-result-medicolegal-concerns (accessed 5 Jun 2021).

8 Bismark MM, Spittal MJ, Plueckhahn TM, et al. Mandatory reports of concerns about the health, performance and conduct of health practitioners. The Medical Journal of Australia 2014;201:399-403. doi:10.5694/mja14.00210
9 Dutheil F, Aubert C, Pereira B, et al. Suicide among physicians and health-care workers: A systematic review and meta-analysis. PLOS ONE 2019;14: 0226361. doi:10.1371/journal.pone.0226361

10 Milner AJ, Maheen H, Bismark MM, et al. Suicide by health professionals: a retrospective mortality study in Australia, 2001-2012. The Medical Journal of Australia 2016;205:260-5. doi:10.5694/mja15.01044

11 Casey D, Choong KA. Suicide whilst under GMC's fitness to practise investigation: Were those deaths preventable? Journal of Forensic and Legal Medicine 2016;37:22-7. doi:10.1016/j.jflm.2015.10.002

12 Wardle JL, Sibbritt D, Broom A, et al. Is Health Practitioner Regulation Keeping Pace with the Changing Practitioner and Health-Care Landscape? An Australian Perspective. Front Public Health 2016;4.

doi:10.3389/fpubh.2016.00091

13 Bawa-Garba case has left profession shaken and stirred | The BMJ.

https://www.bmj.com/content/360/bmj.k45 6 (accessed 5 Jun 2021).

14 The Bawa-Garba case | The BMJ. https://www.bmj.com/bawa-garba (accessed 21 Jan 2020).

15 Singhal P, Pandya B. Beyond BAWA-GARBA: Sushruta Journal of Health Policy \& Opinion 2018;11:20-1. doi:10.38192/11.1.8

16 Understanding the stresses and strains of being a doctor | The Medical Journal of Australia. https://www.mja.com.au/journal/2004/181/ 7/understanding-stresses-and-strains-beingdoctor (accessed 5 Jun 2021).

17 Australian Psychiatrists Today: Proud of their Profession but Stressed and Apprehensive about the Future - Joseph M Rey, Garry Walter, Michael Giuffrida, 2004.

https://journals.sagepub.com/doi/10.1080/j. 1440-1614.2004.01320.x (accessed 5 Jun 2021). 